\title{
Case series of complex therapy of hemoblastosis in the context of COVID-19
}

\author{
Vadim Kemaykin $^{1}$, Ruzal Vildanova ${ }^{1}$, Jamilya Saparbay ${ }^{2}$, Azat Karabekov ${ }^{1}$, Zhandos Burkitbayev ${ }^{3}$ \\ ${ }^{I}$ Department of Oncohematology and Bone Marrow Transplantation, National Research Oncology Center, Republic of Kazakhstan \\ ${ }^{2}$ Department of Gastroenterology, Hepatology and Organ Transplantation, National Research Oncology Center, Republic of Kazakhstan \\ ${ }^{3}$ Chairman of the Board, National Research Oncology Center, Republic of Kazakhstan
}

Received: 2020-11-09.

Accepted: 2020-11-22

This work is licensed under a Creative Commons Attribution 4.0 International License

J Clin Med Kaz 2021; 18(2):75-78

Corresponding author:

Jamilya Saparbay.

E-mail: dzhamilyasaparbay@gmail.com;

ORCID: 0000-0002-8478-5452

\section{Abstract}

Introduction: The COVID-19 pandemic is a problem of a global scale, and it has an importance in modern oncology and hematology. Patients with cancer are at high risk of getting infected with COVID-19. Diagnosis of coronavirus infection in such patients entails the cancellation of chemotherapy for an indefinite period. A break between courses of treatment, as well as a complete cancellation of therapy, can increase the mortality of patients from oncohematological diseases.

Material and methods: From June to October 2020, based on the Oncohematological Department of the National Research Oncology Center LLP, 19 patients were treated, of which: men - 11, women - 8. Average age -43 years (+ -16). Of these, patients with multiple myeloma - 32\%; acute myeloid leukemia - 26\%; acute promyelocytic leukemia - 11\%; acute lymphoblastic leukemia - 26\%; chronic lymphocytic leukemia - 5\%. Treatment was carried out following the protocols for the diagnosis and treatment of hemoblastosis and concomitant COVID-19 infection.

Results: This article presents clinical cases of complex therapy of patients with hemoblastosis and concomitant COVID-19 infection who were treated in a hospital.

Key words: COVID-19, hemoblastosis, multiple myeloma, chemotherapy, autologous stem-cell transplantation, acute myeloid leukemia

\section{Introduction}

The novel coronavirus infection COVID-19 led to an outbreak at the end of December 2019 in the Chinese city Wuhan, which quickly spread around the world. In March 2020, the World Health Organization announced a pandemic of the new coronavirus SARS-CoV-2 COVID-19. Over 40 thousand cases of coronavirus infection and more than 1 million deaths have been registered in the world [1]. In Kazakhstan, the first case of coronavirus infection was registered in March 2020; by October 2020 there are 109623 cases of infection and 1768 deaths [2].

Age and compromised immune systems are predictive factors for COVID-19 infection [3]. Patients with hematological diseases, especially malignant neoplasms, are more susceptible to any infection than the general population due to the obligatory systemic immunosuppression. Immunosuppression and chemotherapy leads to neutropenia, lymphopenia and disorders of the mechanisms of innate and adaptive immunity. Thus, patients with hematologic malignancies are at high risk of infecting with COVID-19 and worse prognosis. These patients can also spread the virus for a longer time due to the increased viral load and delayed elimination of the virus [4].

Currently, there are no guidelines for the treatment of hemoblastosis in the context of COVID-19, recommendations in this direction are based on the opinion and experience of experts [5].

\section{Case presentation Case 1}

A 47-year-old female patient was diagnosed with multiple myeloma. At diagnosis she had pain in the lumbar spine and right shoulder joint. Computed tomography (CT) showed multiple oncolytic lesions of the thoracic spine, sternum, scapula and iliac bones. 
In September 2019, the patient was admitted to our hospital. Laboratory findings showed the existence of Paraprotein (lambda chains) and Bens-Jones protein in urine. Further examination showed $23 \%$ of bone marrow infiltration with plasma cells. The patient also had hypercalcemia, renal failure and anemia. Together with bone disease symptoms comprised CRAB syndrome (hypercalcemia;renal failure; anemia; bone lesions). Considering CRAB syndrome, laboratory findings and bone marrow infiltration the patient was diagnosed with multiple myeloma [6].

The patient underwent 5 courses of BortezomibCyclophosphamid-Dexamethasone (VCD) chemotherapy scheme. Control restaging showed that according to the International Myeloma Working Group (IMWG) the response was not achieved, plasma cell infiltration remained on the same level- 22\% [7]. The patient underwent second-line treatment with 2 courses Talidomid-Cyclophosphamid-Dexamethasone (TCD). The disease was resistant to conventional therapies. Therefore, the treatment was continued with 2 courses of Dexamethasonelenalidomide-cisplatine-doxorubicin-cyclophosphamidetoposide (DR-PACE) scheme [8,9]. However, despite the intensive courses, bone marrow infiltration was $28 \%$. Further treatment was discussed; complete absence of any response to previous three schemes of chemotherapy and possible toxicity of repeated chemotherapy were taken in account. Considering these factors, it was decided to perform autologous hematopoietic stem cell transplantation (Auto-SCT).

The patient underwent "mobilization" of peripheral hematopoietic stem cells (HSCs) with Etoposide $(375 \mathrm{mg} / \mathrm{m} 2)$ and granulocyte colony stimulating factor (G-CSF), 9.23x106 CD34 + cells / $\mathrm{kg}$ were collected. The patient was discharged for

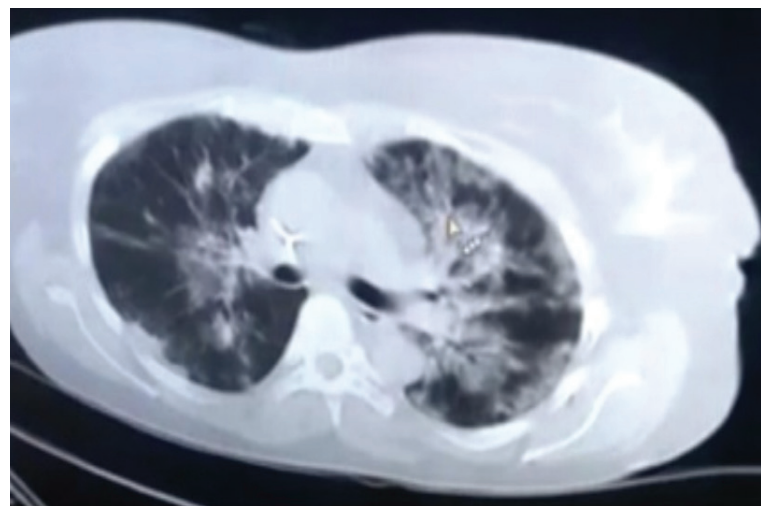

Computed tomography of the chest organs 27.07.2020 the recovery period. On July 2, the patient underwent high-dose melphalan (HD-MEL) (200 mg/m2) supported by autologous hematopoietic stem cells transplantation $(5.58 \mathrm{million} / \mathrm{kg})$. On day 17 after Auto-SCT, the patient developed febrile neutropenia and symptoms of pulmonary aspergillosis. Antifungal therapy with voriconazole; antibacterial therapy with piperacillintazobactam were prescribed. Non-engraftment of the bone marrow $(0.5$ thousand/ $\mu l)$ was recorded on day 25 after AutoSCT. The patient develop acute respiratory failure, respiratory rate (RR) up to 36 per minute, saturation ( $\mathrm{SpO} 2)$ with oxygen insufflation of 10 liters per minute - 90-91\%, fever, unproductive cough. Computed tomography of the lungs showed subtotal infiltration (involvement of the both lung parenchyma was about $60 \%$ ). The nasopharyngeal smear was taken, for SARS-CoV-2 PCR test performance. Due to respiratory failure, the patient was transferred to intensive care unit. According to the national protocols of COVID-19 infection treatment, anticoagulant therapy, glucocorticosteroid therapy (GCS) with dexamethasone $12 \mathrm{mg}$ per day, as well as NIV (non-invasive ventilation of the lungs) in CPAP mode with $\mathrm{FiO} 2=65 \%$, prone body position was applied. On day 28 after transplantation, SARS-CoV-2 RNA from a nasopharyngeal smear was detected by PCR. Bone marrow non-engraftment significantly worsened the course of the disease. 1 week after transfer to intensive care unit and complex therapy initiation the patient's condition significantly improved.

Control computed tomography of the chest organs showed positive dynamics, a decrease in the size of infiltration in the lungs, the volume of lung lesions was $40 \%$ (Figure 1 ), recovery of blood parameters (neutrophilic engraftment was noted) (Table 1).

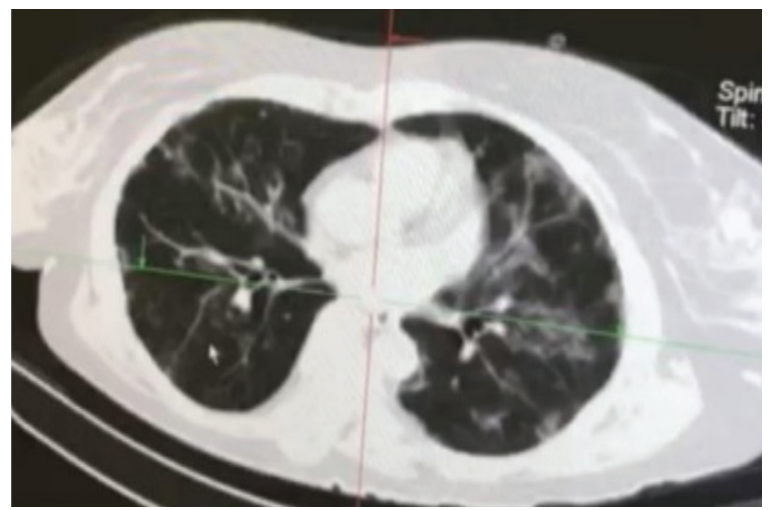

Computed tomography of the chest organs 07.08.2020

Figure 1. Bilateral polysegmental pneumonia. Foci of destruction of the spinal column and bones of the thoracic segment (myeloma). CT signs of bilateral interstitial pneumonia, probably of viral etiology. Foci of destruction of the thoracic vertebra and chest (myeloma) In comparison with the CT scan from 07/27/2020, there is a decrease in the size of infiltration in the lungs

Table 1

Changes in peripheral blood parameters by day from the moment of autologous bone marrow transplantation. Against the background of the complex therapy of hemoblastosis and COVID-19, an increase in the level of leukocytes is observed and bone marrow engraftment is noted.

\begin{tabular}{llllllllll} 
Characteristic & 14POD & 18POD & 27POD & 29POD & 31POD & 34POD & 36POD & 57POD & 61POD \\
\hline WBC/l & 0.1 & 1.6 & 0.6 & 0.3 & 0.1 & 0.3 & 1.7 & 2.4 & 2.8 \\
HB g/l & 69 & 65 & 101 & 95 & 85 & 74 & 59 & 62 & 67 \\
PLT/l & 11 & 5 & 44 & 20 & 68 & 16 & 34 & 42 & 56
\end{tabular}

However, despite the clinical improvement, PCR test showed persistence of SARS-CoV-2 RNA. The patient was transferred back to the ward. On day +62 after Auto-HSCT RNA SARS-CoV-2 from a nasopharyngeal smear was not detected.
In this regard, at day 64 after Auto-HSCT, the patient was discharged from the hospital with improvement, with a relief of signs of coronavirus infection. 


\begin{tabular}{lllllll} 
Characteristic & 1POD & 6POD & 13POD & 21POD & 24POD & 36POD \\
\hline WBC/l & 45 & 4 & 0.4 & 0.6 & 0.8 & 1.3 \\
HB g/l & 50 & 62 & 63 & 37 & 67 & 50 \\
PLT/l & 11 & 23 & 17 & 5 & 17 & 32
\end{tabular}

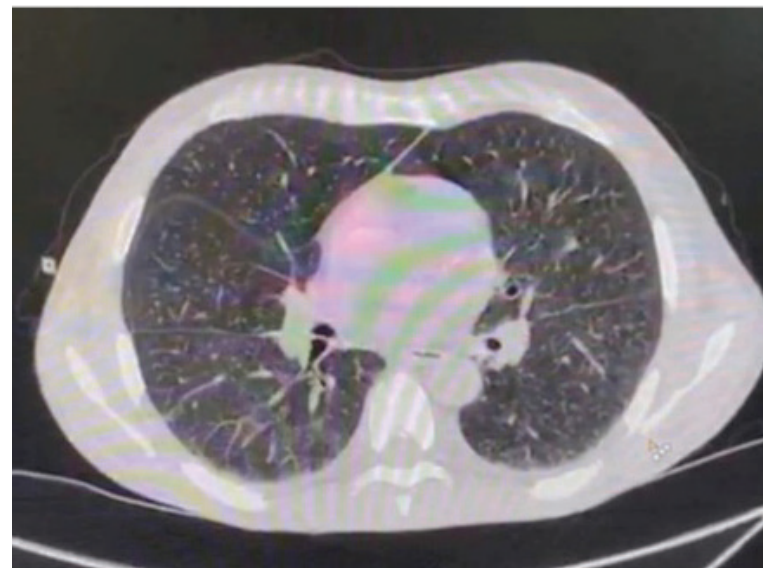

Computed tomography of the chest organs $\mathbf{2 1 . 0 8 . 2 0 2 0}$

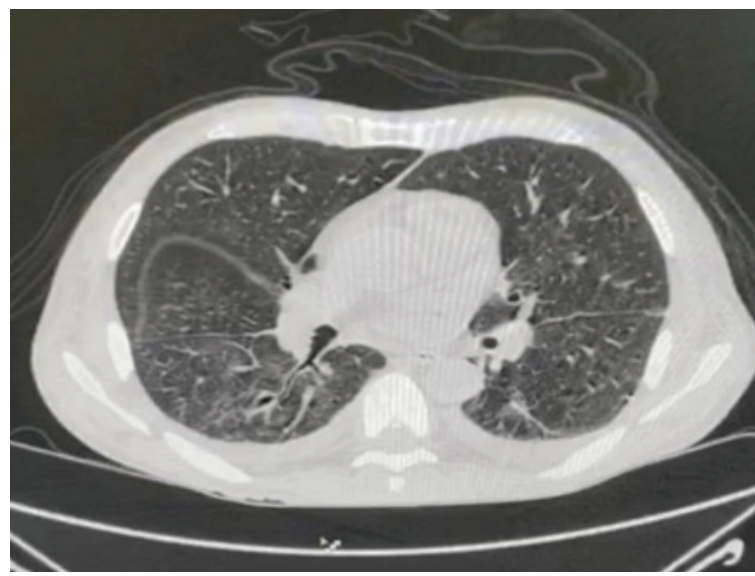

Computed tomography of the chest organs $\mathbf{2 8 . 0 8 . 2 0 2 0}$

Figure 2. Figure 2 Bilateral polysegmental pneumonia, bilateral hydrothorax. In comparison with the CT scan of 08.21 . there is an increase in the degree of infiltration and the development of bilateral hydrothorax.

\section{Case 2}

A 54-year-old patient was diagnosed with acute myeloid leukemia complicated by coronavirus infection, which debuted with changes according to the general blood count in the form of severe anemia, thrombocytopenia, leukocytosis, and blastemia. SARS-CoV-2 RNA was detected from a nasopharyngeal smear.

In August 2020, the patient was admitted to our center. Laboratory examination showed decrease in hemoglobin and platelets, increased level of leukocytes, the presence of tumor cells (blasts) in the peripheral blood and bone marrow. Computed tomography of the lungs showed ground-glass opacification. The patient was diagnosed with acute myeloblastic leukemia with a concomitant COVID-19 [10].

The patient underwent a chemotherapy course according to the "7+3DNR $60 \mathrm{mg} / \mathrm{m} 2 "$ scheme. For COVID-19 pneumonia, the patient received treatment with dexamethasone $8 \mathrm{mg}$ per day, heparin therapy, as well as non-invasive ventilation (NIV) in the CPAP mode with $\mathrm{FiO} 2=70 \%$, and prone body position.

The patient had persistent fever, an increase in C-reactive protein (CRP) and procalcitonin levels, the condition was regarded as febrile neutropenia. Antibacterial therapy with piperacillintazobactam $4.5 \mathrm{~g} 4$ times a day intravenously was prescribed. The patient's condition improved, CRP level was normal. On day 12 after chemotherapy the patient again developed repeated fever, increased CRP level. It was decided to change the tazobactam to meropenem $1.0 \mathrm{~g} 3$ times a day intravenously. Correction of the antibacterial therapy resulted in patient's condition improvement. Respiratory function recovered. Control PCR COVID-19 on day 19 after chemotherapy was negative. However, computed tomography showed slight increase in lung infiltration (Figure 2).

Bone marrow punctate analysis showed that blasts was $50 \%$, remission was not achieved. It was decided to continue the chemotherapy. The patient did not give his consent for further treatment. The patient was discharged from the hospital.

\section{Discussion}

According to Chinese researchers, patients with cancer have worse outcomes from COVID-19, which suggests that this group should be given more attention. Three main strategies are proposed for patients with cancer during the COVID-19 pandemic and in the event of severe infectious complications. First, in endemic regions, consideration should be given to deferring chemotherapy and routine hospitalization. Secondly, more stringent personal protection measures for cancer patients should be envisaged. Third, more intensive monitoring or treatment should be considered when patients are infected with SARS-CoV-2 [11].

Between June 2020 and October 2020 over 200 patients with hemoblastoses were admitted to our hospital, 19 patients had concomitant COVID-19. Among those 19, 11 (57.9\%) were male, $8(42.1 \%)$ were female; the mean age was 43 years. $32 \%$ with multiple myeloma; acute myeloid leukemia - $26 \%$; acute promyelocytic leukemia - 11\%; acute lymphoblastic leukemia - 26\%; chronic lymphocytic leukemia - 5\%. Treatment was carried out following the protocols for the diagnosis and treatment of hemoblastosis and concomitant COVID-19 infection [12]. Among 19 patients with hemoblastosis and concomitant COVID-19 infection, all patients developed COVID-19 pneumonia. Among them, 13 patients had mild symptoms of coronavirus infection, 6 patients required treatment in intensive care unit. Overall, 2 patients were intubated and 4 patients required non-invasive lung ventilation. In all cases, CT scanning showed ground-class opacification with different size of infiltration. In one case patient showed reduction of viral load after treatment, however in majority of observed cases SARSCoV RNA persisted even after clinical improvement. 
In our center, we have chosen the tactics of conducting complex therapy for patients with hemoblastosis and hematopoietic depression with COVID-19 without interrupting chemotherapy. According to our preliminary observation, in majority of patients (13 patients) chemotherapy does not worsen the course of COVID-19 and does not increase early mortality after chemotherapy. We noted that the SARS-CoV RNA persisted in the patient's blood tests even after clinical improvement. Thus, this group of patients should be isolated for longer period in comparison with group of patients with noncompromised immune system.

Currently, there is not enough research regarding superiority one or another treatment approach of patients with hemoblastosis and coronavirus infection. In our experience, we evaluated the effect of complex therapy in patients with hematopoietic malignancies and depression of hematopoiesis with COVID-19, without interrupting chemotherapy. The proposed protocol will presumably reduce the risks of recurrence and resistance to the chemotherapy. Further research and larger case series are required for development effective treatment approaches of the patients with hematological malignancies and concominant COVIID-19.

\section{Abbreviations:}

COVID-19 - coronavirus disease 2019

SARS-CoV-2 - severe acute respiratory syndrome related coronavirus 2
CT - computed tomography

NROC - National Research Oncology Center LLP

CRAB - mnemonic abbreviation: hyperCalcemia; Renal insufficiency; Anemia; Bone lesions

CTX - chemotherapy

IMWG - International Myeloma Working Group

Auto-SCT - autologous stem-cell transplantation

BMT - bone marrow transplantation

GCS - glucocorticosteroid

NIV - non-invasive ventilation of the lungs

PCR - polymerase chain reaction

ABT - antibiotic therapy

CRP - c-reactive protein

TCD- Thalidomide-Cyclophosphamide-Dexametazone (chemotherapy scheme)

VCD- Bortezomib-Cyclophosphamide-Dexamethasone (chemotherapy scheme)

HD-MEL- High-dose melphalan

Disclosures: There is no conflict of interest for all authors.

Acknowledgements: We would like to thank the patient for allowing us to share the details of treatment in this paper.

Funding information: None.

\section{References}

1. Worldometer, 2020. COVID-19 Coronavirus Pandemic. URL: https://www.worldometers.info/coronavirus/v. Accessed October 20, 2020 .

2. Kazinform, 2020. COVID-19 Epidemiya koronavirusa. URL: https://www.coronavirus2020.kz. Accessed October 20, 2020.

3. Zhu WJ, Wang J, and He XH, et al. The differential diagnosis of pulmonary infiltrates in cancer patients during the outbreak of the 2019 novel coronavirus disease. Zhonghua Zhong Liu Za Zhi. 2020; 42(0):E008 PMID; DOI: 10.3760/cma.j.cn112152-20200303-00166

4. Robert Weinkove, Zoe K McQuilten, Jonathan Adler, Meera R Agar, Emily Blyth, et al. Managing haematology and oncology patients during the COVID-19 pandemic: interim consensus guidance. Wiley Public Health Emergency Collection. 2020; 212(10):481-489 DOI: $10.5694 / \mathrm{mja} 2.50607$

5. G.Curigliano, S.Banerjee, A.Cervantes, M.C.Garassino, P.Garrido, et al. Managing cancer patients during the COVID-19 pandemic: an ESMO multidisciplinary expert consensus. 2020. DOI: 10.1016/j.annonc.2020.07.010

6. Rajkumar SV. Updated Diagnostic Criteria and Staging System for Multiple Myeloma. Am Soc Clin Oncol Educ Book. 2016; 35:e41823. DOI: $10.1200 / E D B K \_159009$. PMID: 27249749. DOI: 10.1200/EDBK_159009

7. National Comprehensive Cancer Network www.nccn.org

8. Dadacaridou M, Papanicolaou X, Maltesas D, Megalakaki C, Patos P, Panteli K, Repousis P, Missouri-Mentzikof C. Dexamethasone, cyclophosphamide, etoposide, and cisplatin (DCEP) for relapsed or refractory multiple myeloma patients PMID: 17436400

9. Srikanth M, Davies FE, Wu P, et al. Survival and outcome of blastoid variant myeloma following treatment with the novel thalidomide containing regime DT-PACE. Eur J Haematol. 2008; 81:432-436 DOI: 10.1111/j.1600-0609.2008.01131.x

10. Arber DA, Orazi A, Hasserjian R, et al. The 2016 revision to the World Health Organization classification of myeloid neoplasms and acute leukemia. Blood. 2016; 127(20):2391-405. DOI: 10.1182/blood-2016-03-643544

11. Liang W, Guan W, Chen R, et al. Cancer patients in SARS-CoV-2 infection: a nationwide analysis in China. Lancet Oncol. 2020; 21(3):335-337. DOI:10.1016/S1470-2045(20)30096-6

12. Clinical guidelines of Kazakhstan Health Care Ministry "Coronovirus infection treatment"- 10-th edition with changes from 15.07.2020. 